\title{
Lyapunov functions and strict stability of Caputo fractional differential equations
}

\author{
Ravi Agarwal ${ }^{1,2}$, Snezhana Hristova ${ }^{3 *}$ and Donal O'Regan ${ }^{4}$
}

"Correspondence: snehri@gmail.com

${ }^{3}$ Plovdiv University, Tzar Asen 24, Plovdiv, 4000, Bulgaria

Full list of author information is available at the end of the article

\begin{abstract}
One of the main properties studied in the qualitative theory of differential equations is the stability of solutions. The stability of fractional order systems is quite recent. There are several approaches in the literature to study stability, one of which is the Lyapunov approach. However, the Lyapunov approach to fractional differential equations causes many difficulties. In this paper a new definition (based on the Caputo fractional Dini derivative) for the derivative of Lyapunov functions to study a nonlinear Caputo fractional differential equation is introduced. Comparison results using this definition and scalar fractional differential equations are presented, and sufficient conditions for strict stability and uniform strict stability are given. Examples are presented to illustrate the theory.
\end{abstract}

MSC: 34A34; 34A08; 34D20

Keywords: strict stability; Lyapunov functions; Caputo derivatives; fractional differential equations

\section{Introduction}

One of the main problems in the qualitative theory of differential equations is stability of solutions. However, the usual stability concepts do not give any information concerning the rate of decay of solutions, and hence are not strict concepts. As a result, strict stability was defined, and criteria for such notions were discussed (see, for example, [1-5]).

Fractional differential equations play an important role not only in mathematics but also in physics, control systems, dynamical systems, engineering and in particular in mathematical modeling of many natural physical phenomena. For example, fractional derivatives are used in modeling mechanical and electrical properties of real materials, in the description of properties of gases, liquids and rocks, and in many other fields (see, for example, $[6,7])$.

The stability of fractional order systems is quite recent. The analysis on stability of fractional differential equations is more complicated than classical differential equations since fractional derivatives are nonlocal and have weakly singular kernels. Recently, in [8] an overview on stability results of fractional differential equations is given. For nonlinear fractional differential equations, there are several approaches in the literature to study stability, one of which is the Lyapunov approach. The investigations in the literature via Lyapunov functions could be divided into two main groups:

(c) 2015 Agarwal et al. This article is distributed under the terms of the Creative Commons Attribution 4.0 International License (http://creativecommons.org/licenses/by/4.0/), which permits unrestricted use, distribution, and reproduction in any medium, provided you give appropriate credit to the original author(s) and the source, provide a link to the Creative Commons license, and indicate if changes were made. 
- continuously differentiable Lyapunov functions (see, for example, the papers [9-15]). Different types of stability are discussed using the Caputo derivative of Lyapunov functions which depends significantly on the unknown solution of the fractional equation;

- continuous Lyapunov functions (see, for example, the papers [16-18]) in which the authors use the derivative of a Lyapunov function which is similar to the Dini derivative of Lyapunov functions.

In this paper the strict stability of nonlinear nonautonomous Caputo fractional differential equations is defined and studied using continuous Lyapunov functions. The Caputo fractional Dini derivative of a Lyapunov function is defined in an appropriate way. Note that this type of derivative is introduced in [19] and used to study the stability and asymptotic stability of Caputo fractional differential equations. Comparison results using this definition and scalar fractional differential equations are presented, and sufficient conditions for strict stability and uniform strict stability are obtained.

The manuscript is organized as follows. In Section 2 some preliminaries of fractional calculus are mentioned. Section 3 presents basic definitions concerning strict stability and the new definition of the Caputo fractional Dini derivative of Lyapunov functions among the nonlinear fractional differential equations. In Section 4 some comparison results are given. Section 5 presents some sufficient conditions for strict stability and uniform strict stability.

\section{Notes on fractional calculus}

Fractional calculus generalizes the derivative and the integral of a function to a non-integer order [20,21], and there are several definitions of fractional derivatives and fractional integrals. In engineering, the fractional order $q$ is often less than 1 , so we restrict our attention to $q \in(0,1)$.

(1) The Riemann-Liouville (RL) fractional derivative of order $q \in(0,1)$ of $m(t)$ is given by (see, for example, Section 1.4.1.1 [22], or [20])

$$
{ }_{t_{0}}^{R L} D^{q} m(t)=\frac{1}{\Gamma(1-q)} \frac{d}{d t} \int_{t_{0}}^{t}(t-s)^{-q} m(s) d s, \quad t \geq t_{0},
$$

where $\Gamma(\cdot)$ denotes the gamma function.

(2) The Caputo fractional derivative of order $q \in(0,1)$ is defined by (see, for example, Section 1.4.1.3 [22])

$$
{ }_{t_{0}}^{c} D^{q} m(t)=\frac{1}{\Gamma(1-q)} \int_{t_{0}}^{t}(t-s)^{-q} m^{\prime}(s) d s, \quad t \geq t_{0} .
$$

The Caputo and Riemann-Liouville formulations coincide when $m\left(t_{0}\right)=0$. The properties of the Caputo derivative are quite similar to those of ordinary derivatives. Also, the initial conditions of fractional differential equations with the Caputo derivative have a clear physical meaning, and, as a result, the Caputo derivative is usually used in real applications.

(3) The Grunwald-Letnikov fractional derivative is given by (see, for example, Section 1.4.1.2 [22])

$$
{ }_{t_{0}}^{G L} D^{q} m(t)=\lim _{h \rightarrow 0+} \frac{1}{h^{q}} \sum_{r=0}^{\left[\frac{t-t_{0}}{h}\right]}(-1)^{r}(q C r) m(t-r h), \quad t \geq t_{0},
$$




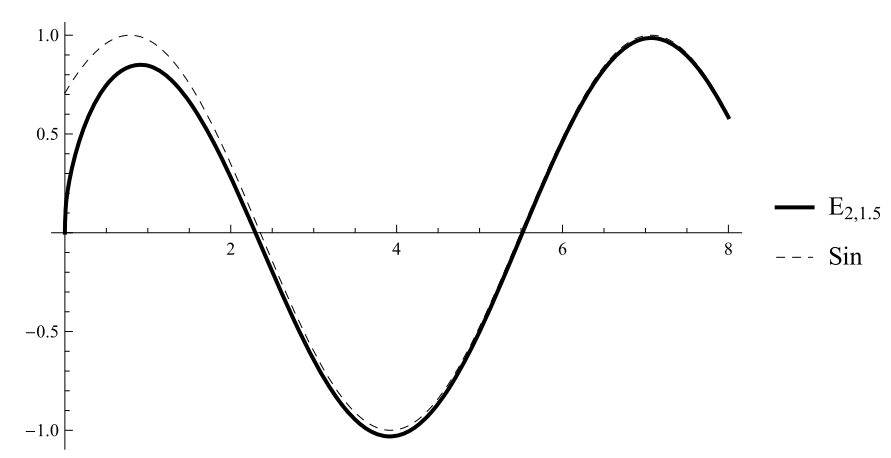

Figure 1 Graphs of $t^{1-q} E_{2,2-q}\left(-t^{2}\right)$ and $\sin \left(t+q \frac{\pi}{2}\right)$ for $q=0.5$.

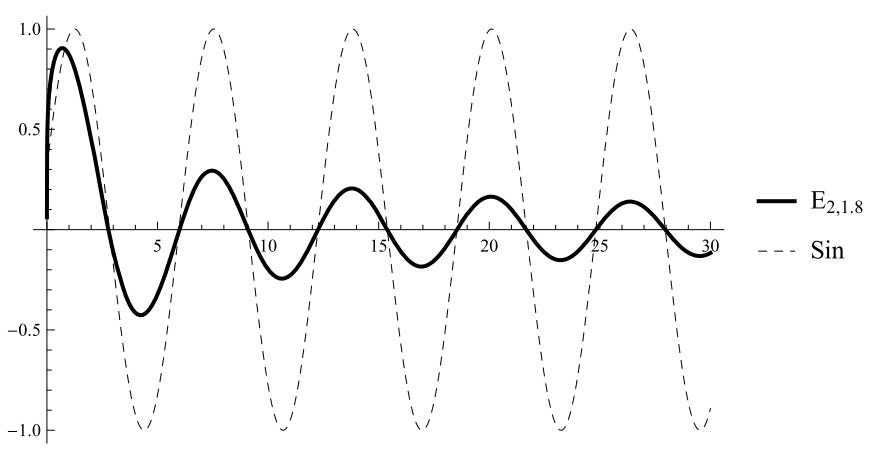

Figure 2 Graphs of $t^{1-q} E_{2,2-q}\left(-t^{2}\right)$ and $\sin \left(t+q \frac{\pi}{2}\right)$ for $q=0.2$.

or

$$
{ }_{t_{0}}^{G L} D^{q} m(t)=\lim _{h \rightarrow 0+} \frac{1}{h^{q}} \sum_{r=0}^{\left[\frac{t-t_{0}}{h}\right]}(-1)^{r} \frac{\Gamma(1+q)}{r ! \Gamma(1+q-r)} m(t-r h), \quad t \geq t_{0},
$$

and the Grunwald-Letnikov fractional Dini derivative by

$$
{ }_{t_{0}}^{G L} D_{+}^{q} m(t)=\limsup _{h \rightarrow 0+} \frac{1}{h^{q}} \sum_{r=0}^{\left[\frac{t-t_{0}}{h}\right]}(-1)^{r}(q C r) m(t-r h), \quad t \geq t_{0}
$$

where $q C r$ are the binomial coefficients and $\left[\frac{t-t_{0}}{h}\right]$ denotes the integer part of the fraction $\frac{t-t_{0}}{h}$.

Example 1 The behavior of the derivative depends significantly on its order $q$. For example, consider ${ }_{0}^{G L} D^{q} \sin (t)=t^{1-q} E_{2,2-q}\left(-t^{2}\right)$. For $q=0.5$, the derivative is not periodic, but it converges to the periodic function $\sin \left(t+q \frac{\pi}{2}\right.$ ) (see Figure 1). For $q=0.2$, the behavior of the fractional derivative is totally different (see Figure 2).

The relation between the two types of fractional derivatives is given by the equality ${ }_{t_{0}}^{c} D^{q} m(t)={ }_{t_{0}}^{R L} D^{q}\left[m(t)-m\left(t_{0}\right)\right]$. 
Proposition 1 (Theorem 2.25 [23]) Let $m \in C^{1}\left[t_{0}, b\right]$. Then, for $t \in\left(t_{0}, b\right],{ }_{t_{0}}^{G L} D^{q} m(t)=$ ${ }_{t_{0}}^{R L} D^{q} m(t)$.

Also, according to Lemma 3.4 [23], the equality ${ }_{t_{0}}^{c} D_{t}^{q} m(t)={ }_{t_{0}}^{R L} D_{t}^{q} m(t)-m\left(t_{0}\right) \frac{\left(t-t_{0}\right)^{-q}}{\Gamma(1-q)}$ holds.

From the relation between the Caputo fractional derivative and the Grunwald-Letnikov fractional derivative, using (2) we define the Caputo fractional Dini derivative as

$$
{ }_{t_{0}}^{c} D_{+}^{q} m(t)={ }_{t_{0}}^{G L} D_{+}^{q}\left[m(t)-m\left(t_{0}\right)\right]
$$

i.e.,

$$
{ }_{t_{0}}^{c} D_{+}^{q} m(t)=\limsup _{h \rightarrow 0+} \frac{1}{h^{q}}\left[m(t)-m\left(t_{0}\right)-\sum_{r=1}^{\left[\frac{t-t_{0}}{h}\right]}(-1)^{r+1}(q C r)\left(m(t-r h)-m\left(t_{0}\right)\right)\right] .
$$

Definition 1 ([16]) We say $m \in C^{q}\left(\left[t_{0}, T\right], \mathbb{R}^{n}\right)$ if $m(t)$ is differentiable (i.e., $m^{\prime}(t)$ exists), the Caputo derivative ${ }_{t_{0}}^{c} D^{q} m(t)$ exists and satisfies (1) for $t \in\left[t_{0}, T\right]$.

Remark 1 If $m \in C^{q}\left(\left[t_{0}, T\right], \mathbb{R}^{n}\right)$, then ${ }_{t_{0}}^{c} D_{+}^{q} m(t)={ }_{t_{0}}^{c} D^{q} m(t)$

\section{Statement of the problem}

Consider the initial value problem (IVP) for the system of fractional differential equations (FrDE) with a Caputo derivative for $0<q<1$,

$$
{ }_{t_{0}}^{c} D^{q} x=f(t, x), \quad x\left(t_{0}\right)=x_{0},
$$

where $x, x_{0} \in \mathbb{R}^{n}, f \in C\left[\mathbb{R}_{+} \times \mathbb{R}^{n}, \mathbb{R}^{n}\right], f(t, 0) \equiv 0, t_{0} \geq 0$.

We will assume in the paper that the function $f \in C\left[\mathbb{R}_{+} \times \mathbb{R}^{n}, \mathbb{R}^{n}\right]$ is such that for any initial data $\left(t_{0}, x_{0}\right) \in \mathbb{R}_{+} \times \mathbb{R}^{n}$, FrDE (4) has a solution $x\left(t ; t_{0}, x_{0}\right) \in C^{q}\left(\left[t_{0}, \infty\right), \mathbb{R}^{n}\right)$. Note that some sufficient conditions for global existence of solutions of (4) are given in [17, 23, 24 .

The goal of the paper is to study strict stability of FrDE (4). Strict stability for fractional equations is studied in [25], but the definitions and conditions are not clear. Now we will define strict stability for fractional equations following the idea for ordinary differential equations (see, for example, [3]).

Definition 2 The zero solution of system FrDE (4) is said to be

- strictly stable if for given $\epsilon_{1}>0$ and $t_{0} \in \mathbb{R}_{+}$there exists $\delta_{1}=\delta_{1}\left(t_{0}, \epsilon_{1}\right)>0$ such that for any initial point $x_{0} \in \mathbb{R}^{n}$ the inequality $\left\|x_{0}\right\|<\delta_{1}$ implies $\left\|x\left(t ; t_{0}, x_{0}\right)\right\|<\epsilon_{1}, t \geq t_{0}$, and for any $\delta_{2}=\delta_{2}\left(t_{0}, \epsilon_{1}\right), \delta_{2} \in\left(0, \delta_{1}\right]$ there exists $\epsilon_{2}=\epsilon_{2}\left(t_{0}, \delta_{2}\right), \epsilon_{2} \in\left(0, \delta_{2}\right]$ such that the inequality $\delta_{2}<\left\|x_{0}\right\|$ implies $\epsilon_{2}<\left\|x\left(t ; t_{0}, x_{0}\right)\right\|$ for $t \geq t_{0}$ where $x\left(t ; t_{0}, x_{0}\right)$ is a solution of the IVP for FrDE (4);

- uniformly strictly stable if for any given $\epsilon_{1}>0$ there exists $\delta_{1}=\delta_{1}\left(\epsilon_{1}\right)>0$ such that for any initial time $t_{0} \in \mathbb{R}_{+}$and any initial point $x_{0} \in \mathbb{R}^{n}$ the inequality $\left\|x_{0}\right\|<\delta_{1}$ implies $\left\|x\left(t ; t_{0}, x_{0}\right)\right\|<\epsilon_{1}, t \geq t_{0}$, and for any $\delta_{2} \in\left(0, \delta_{1}\right]$ there exists $\epsilon_{2} \in\left(0, \delta_{2}\right], \varepsilon_{2}=\varepsilon_{2}\left(\delta_{2}\right)$, such that the inequality $\delta_{2}<\left\|x_{0}\right\|$ implies $\epsilon_{2}<\left\|x\left(t ; t_{0}, x_{0}\right)\right\|$ for $t \geq t_{0}$ where $x\left(t ; t_{0}, x_{0}\right)$ is a solution of the IVP for FrDE (4). 


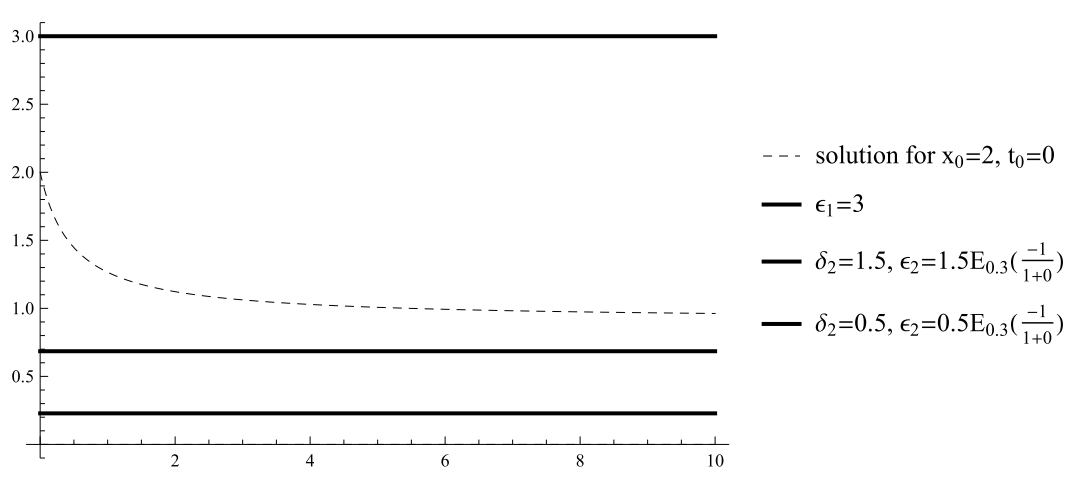

Figure 3 Graph of the solution $x(t)=x_{0} E_{q}\left(\frac{1}{t+1}-\frac{1}{t_{0}+1}\right)$ for $q=0.3, x_{0}=2, t_{0}=0$.

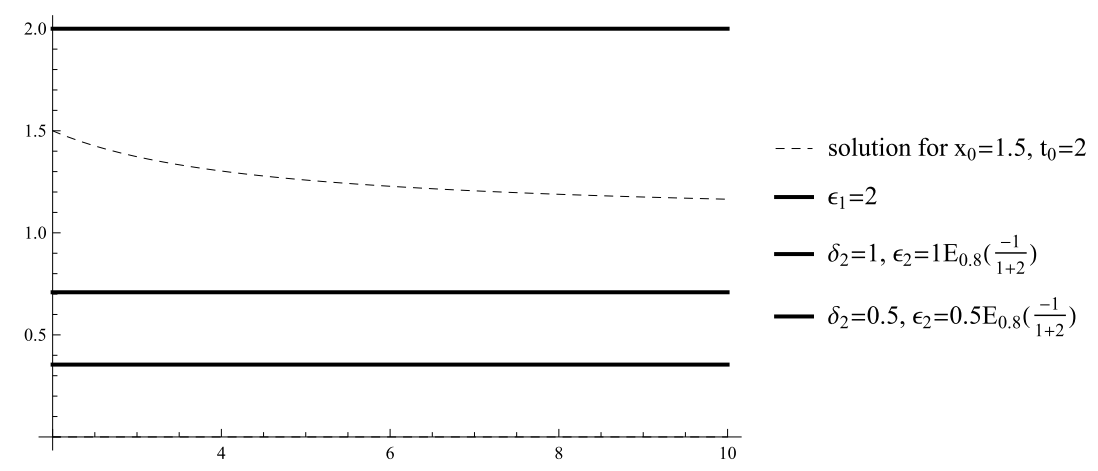

Figure 4 Graph of the solution $x(t)=x_{0} E_{q}\left(\frac{1}{t+1}-\frac{1}{t_{0}+1}\right)$ for $q=0.8, x_{0}=1.5, t_{0}=2$.

Remark 2 The strict stability immediately implies that the zero solution is not asymptotically stable.

Example 2 (Strict stability) Consider the ODE $x^{\prime}=-\frac{1}{(t+1)^{2}} x, x\left(t_{0}\right)=x_{0}$ with a solution $x(t)=x_{0} e^{-\frac{1}{t_{0}+1}} e^{\frac{1}{t+1}}$. Since $1<e^{\frac{1}{t+1}} \leq e$ for $t \in \mathbb{R}_{+}$, it follows that for any $\varepsilon_{1}$ if $\left|x_{0}\right|<\frac{\varepsilon_{1}}{e}=\delta_{1}$ then $x(t)<\varepsilon_{1}$, and for any $\delta_{2} \in\left(0, \delta_{1}\right)$ the inequality $\left|x_{0}\right|>\delta_{2}$ implies $x(t)>\varepsilon_{2}=\delta_{2} e^{-\frac{1}{t_{0}+1}}$. Therefore, the zero solution of the considered ODE is strictly stable.

Now consider a Caputo fractional differential equation with a solution given by $x(t)=$ $x_{0} E_{q}\left(\frac{1}{t+1}-\frac{1}{t_{0}+1}\right)$ where the Mittag-Leffler function (with one parameter) is defined by $E_{q}(z)=\sum_{k=0}^{\infty} \frac{z^{k}}{\Gamma(q k+1)}$. Since $E_{q}\left(-\frac{1}{t_{0}+1}\right)<E_{q}\left(\frac{1}{t+1}-\frac{1}{t_{0}+1}\right) \leq E_{q}(0)=1, t \geq t_{0}$, it follows that for any $\varepsilon_{1}$ if $\left|x_{0}\right|<\delta_{1}=\varepsilon_{1}$ then $x(t)<\varepsilon_{1}$, and for any $\delta_{2} \in\left(0, \delta_{1}\right)$ for $\left|x_{0}\right|>\delta_{2}$ the inequality $x(t)>\varepsilon_{2}=\delta_{2} E_{q}\left(-\frac{1}{t_{0}+1}\right)$ holds. Therefore, the zero solution of the FrDE is strictly stable (see Figure 3 for $q=0.3, t_{0}=0$ and Figure 4 for $q=0.8, t_{0}=2$ ).

In this paper we will use the followings sets:

$$
\begin{aligned}
& \mathcal{K}=\left\{a \in C\left[\mathbb{R}_{+}, \mathbb{R}_{+}\right]: a(u) \text { is strictly increasing and } a(0)=0\right\} \\
& B(\lambda)=\left\{x \in \mathbb{R}^{n}:\|x\| \leq \lambda\right\}, \quad \lambda=\text { const }>0
\end{aligned}
$$


We will use comparison results for scalar Caputo fractional differential equations of the type

$$
{ }_{t_{0}}^{c} D^{q} u=g(t, u) \quad \text { for } t \geq t_{0}
$$

where $u \in \mathbb{R}, g: \mathbb{R}_{+} \times \mathbb{R} \rightarrow \mathbb{R}, g(t, 0) \equiv 0$. Note that (5) with $u\left(t_{0}\right)=u_{0}$ is called the initial value problem (5). Some existence results for (5) are given in [17, 23, 24].

Also, we will discuss a couple of Caputo fractional differential equations of the type

$$
\begin{array}{lll}
{ }_{t_{0}}^{c} D^{q} u=g_{1}(t, u) & \text { for } t \geq t_{0}, & u\left(t_{0}\right)=u_{0}, \\
{ }_{t_{0}}^{c} D^{q} v=g_{2}(t, v) & \text { for } t \geq t_{0}, & v\left(t_{0}\right)=v_{0},
\end{array}
$$

where $u, v \in \mathbb{R}, g_{1}, g_{2}: \mathbb{R}_{+} \times \mathbb{R} \rightarrow \mathbb{R}, g_{i}(t, 0) \equiv 0, i=1,2$. We will assume in the paper that the functions $g_{i}: \mathbb{R}_{+} \times \mathbb{R} \rightarrow \mathbb{R}, i=1,2$, are such that for any initial time $t_{0} \in \mathbb{R}_{+}$and any initial values $u_{0}, v_{0} \in \mathbb{R}$ the couple of scalar $\operatorname{FrDE}(6)$ has a solution $\left(u\left(t ; t_{0}, u_{0}\right), v\left(t ; t_{0}, u_{0}\right)\right)$, $u\left(t ; t_{0}, u_{0}\right), v\left(t ; t_{0}, u_{0}\right) \in C^{q}\left(\left[t_{0}, \infty\right), \mathbb{R}\right)$. In the case of nonuniqueness, we will assume that the first equation of (6) has a maximal solution and the second one has a minimal solution.

Fix $i \in\{1,2\}$. We say that $g_{i}$ satisfies condition (H1) if:

(H1) For any compact interval $\left[t_{0}, T\right] \subset \mathbb{R}_{+}$, there exists a small enough number $L_{t_{0}}>0$ such that for any $|\eta|<L_{t_{0}}$ the IVP for $\operatorname{FrDE}_{t_{0}}^{c} D^{q} u=g_{i}(t, u)+\eta, u\left(t_{0}\right)=u_{0}$ has a solution $u\left(t ; t_{0}, u_{0}, \eta\right) \in C^{q}\left(\left[t_{0}, T\right], \mathbb{R}\right)$ where $u_{0} \in \mathbb{R}$.

We now introduce the strict stability of the couple of Caputo fractional differential equations as follows.

Definition 3 The zero solution of the couple of FrDE (6) is said to be

- strictly stable in couple if for given $\epsilon_{1}>0$ and $t_{0} \in \mathbb{R}_{+}$there exists $\delta_{1}=\delta_{1}\left(t_{0}, \epsilon_{1}\right)>0$ and for any $\delta_{2}=\delta_{2}\left(t_{0}, \epsilon_{1}\right), \delta_{2} \in\left(0, \delta_{1}\right]$ there exists $\epsilon_{2}=\epsilon_{2}\left(t_{0}, \delta_{2}\right), \epsilon_{2} \in\left(0, \delta_{2}\right]$ such that the inequalities $\left|u_{0}\right|<\delta_{1}$ and $\delta_{2}<\left|v_{0}\right|$ imply $\left|u\left(t ; t_{0}, u_{0}\right)\right|<\epsilon_{1}$ and $\epsilon_{2}<\left|v\left(t ; t_{0}, v_{0}\right)\right|$ for $t \geq t_{0}$, where the couple of functions $\left(u\left(t ; t_{0}, u_{0}\right), v\left(t ; t_{0}, u_{0}\right)\right)$ is a solution of the IVP for FrDE (6);

- uniformly strictly stable in couple if for any given $\epsilon_{1}>0$ there exists $\delta_{1}=\delta_{1}\left(\epsilon_{1}\right)>0$ and for any $\delta_{2} \in\left(0, \delta_{1}\right]$ there exists $\epsilon_{2} \in\left(0, \delta_{2}\right], \varepsilon_{2}=\varepsilon_{2}\left(\delta_{2}\right)$, such that for any initial time $t_{0} \in \mathbb{R}_{+}$the inequalities $\left|u_{0}\right|<\delta_{1}$ and $\delta_{2}<\left|v_{0}\right|$ imply $\left|u\left(t ; t_{0}, u_{0}\right)\right|<\epsilon_{1}$ and $\epsilon_{2}<\left|v\left(t ; t_{0}, v_{0}\right)\right|$ for $t \geq t_{0}$, where the couple of functions $\left(u\left(t ; t_{0}, u_{0}\right), v\left(t ; t_{0}, u_{0}\right)\right)$ is a solution of the IVP for FrDE (6).

Example 3 (Uniform strict stability in couple) Consider the couple of Caputo fractional differential equations

$$
\begin{aligned}
& { }_{t_{0}}^{c} D^{q} u=A u \quad \text { for } t \geq t_{0}, \quad u\left(t_{0}\right)=u_{0}, \\
& { }_{t_{0}}^{c} D^{q} v=B v \quad \text { for } t \geq t_{0}, \quad v\left(t_{0}\right)=v_{0} \text {, }
\end{aligned}
$$

where $u, v \in \mathbb{R}, A, B$ are given constants.

The solution of $(7)$ is $\left(u_{0} E_{q}\left(A\left(t-t_{0}\right)^{q}\right), v_{0} E_{q}\left(B\left(t-t_{0}\right)^{q}\right)\right)$, where the Mittag-Leffler function (with one parameter) is defined by $E_{q}(z)=\sum_{k=0}^{\infty} \frac{z^{k}}{\Gamma(q k+1)}$. 


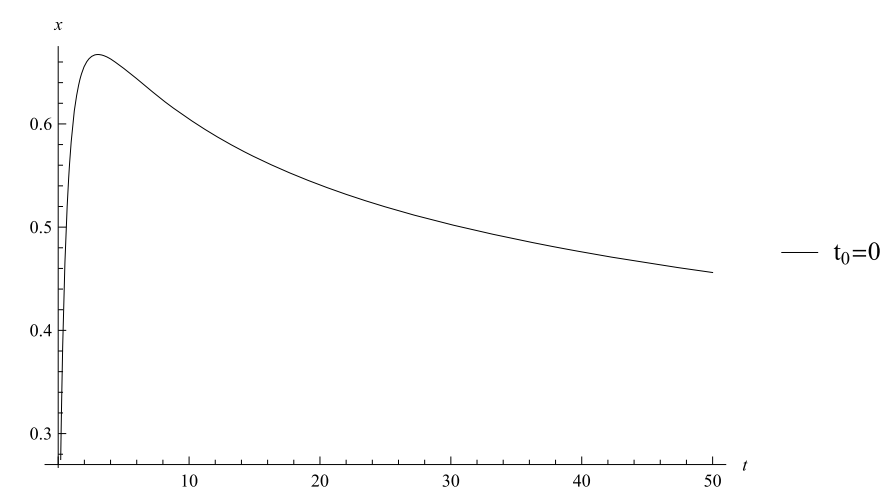

Figure 5 Graph of the function $h\left(t, t_{0}\right), t \geq t_{0}$ for $t_{0}=0$.

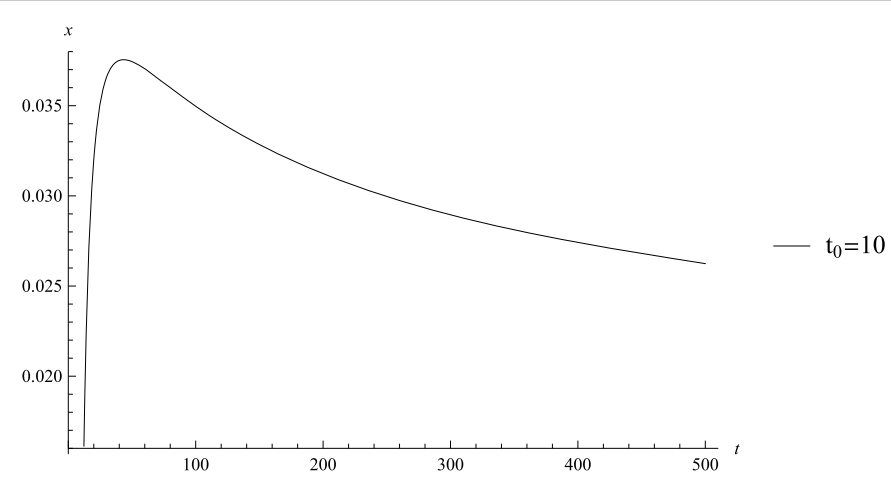

Figure 6 Graph of the function $h\left(t, t_{0}\right), t \geq t_{0}$ for $t_{0}=10$.

Case 1. Let $A<0, B>0$. From the inequalities $0<E_{q}(z) \leq 1$ for $z \leq 0$ and $E_{q}(z) \geq 1$ for $z \geq 0$ it follows that $\left|u_{0} E_{q}\left(A\left(t-t_{0}\right)^{q}\right)\right| \leq\left|u_{0}\right|$ and $\left|v_{0} E_{q}\left(B\left(t-t_{0}\right)^{q}\right)\right| \geq\left|v_{0}\right|$, i.e., the zero solution of the couple of $\operatorname{FrDE}(7)$ is uniformly strictly stable in couple.

Case 2. Let $A, B=0$. Then the solution of ( 7$)$ is $\left(u_{0}, v_{0}\right)$ which shows that the zero solution of the couple of $\operatorname{FrDE}(7)$ is uniformly strictly stable in couple.

Example 4 (Strict stability in couple) Consider the couple of Caputo fractional differential equations

$$
\begin{aligned}
& { }_{t_{0}}^{c} D^{0.2} u=\frac{t}{t+1} \quad \text { for } t \geq t_{0}, \quad u\left(t_{0}\right)=u_{0}, \\
& { }_{t_{0}}^{c} D^{0.2} v=0 \quad \text { for } t \geq t_{0}, \quad v\left(t_{0}\right)=v_{0},
\end{aligned}
$$

where $u, v \in \mathbb{R}$.

The first component of the solution of (8) satisfies the integral equation

$$
u(t)=u_{0}+\int_{t_{0}}^{t} \frac{\frac{1}{1+s}-\frac{s}{(1+s)^{2}}}{(t-s)^{0.2}} d s .
$$

The function $h\left(t, t_{0}\right)=\int_{t_{0}}^{t} \frac{\frac{1}{1+s}-\frac{s}{(1+s)^{2}}}{(t-s)^{0.2}} d s$ has a maximum depending on $t_{0}$ which decreasingly approaches 0 (see Figures $5,6,7,8)$. Then, for any $\varepsilon>0$, there exists $\delta=\delta\left(\varepsilon, t_{0}\right)>0$ 


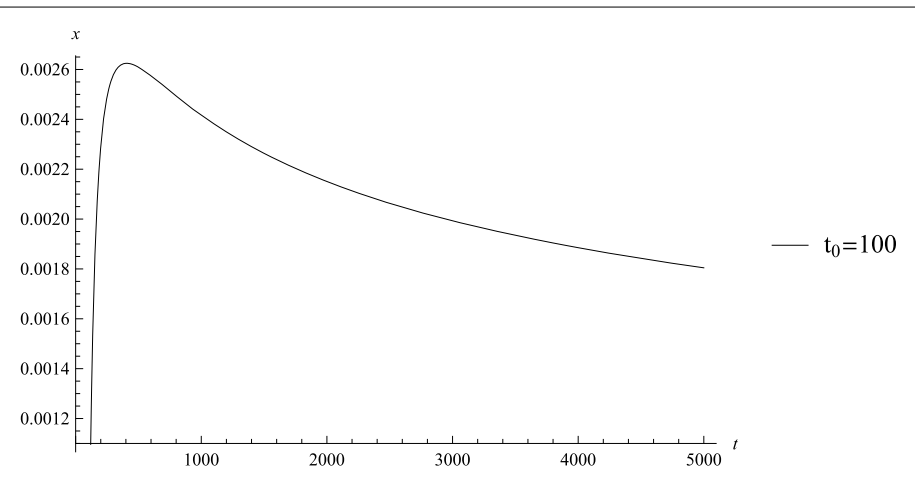

Figure 7 Graph of the function $h\left(t, t_{0}\right), t \geq t_{0}$ for $t_{0}=100$.

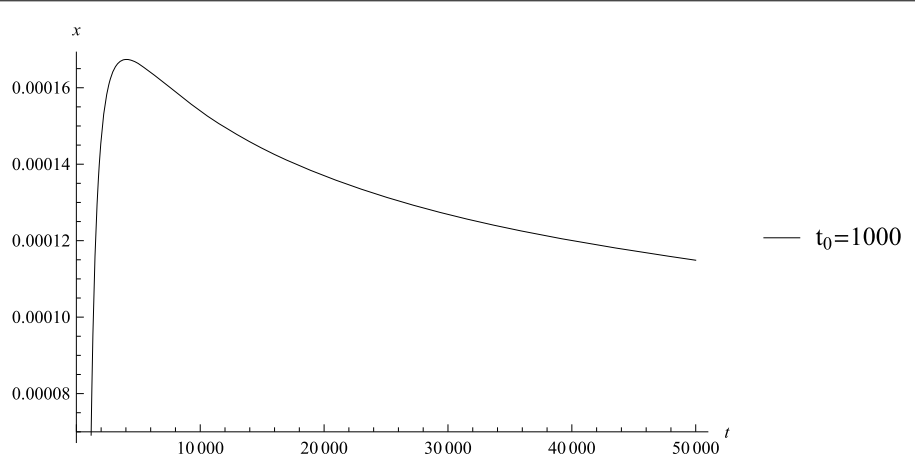

Figure 8 Graph of the function $h\left(t, t_{0}\right), t \geq t_{0}$ for $t_{0}=1,000$.

such that $\left|u_{0}\right|<\delta$ implies $|u(t)|<\varepsilon$. Now $v(t) \equiv v_{0}$, so we see the strict stability in couple of (8).

We now introduce the class $\Lambda$ of Lyapunov-like functions which will be used to investigate the strict stability of the system of $\operatorname{FrDE}(4)$.

Definition 4 Let $t_{0}, T \in \mathbb{R}_{+}: T>t_{0}$, and $\Delta \subset \mathbb{R}^{n}, 0 \in \Delta$. We will say that the function $V(t, x):\left[t_{0}, T\right) \times \Delta \rightarrow \mathbb{R}_{+}$belongs to the class $\Lambda\left(\left[t_{0}, T\right), \Delta\right)$ if $V(t, x) \in C\left[\left[t_{0}, T\right) \times \Delta, \mathbb{R}_{+}\right]$, and it is locally Lipschitzian with respect to its second argument.

Remark 3 In the case when the Lyapunov function does not depend on the time $t$, i.e., $V(t, x)=V(x), V \in C\left[\Delta, \mathbb{R}_{+}\right]$and it is locally Lipschitzian, we denote the class introduced in Definition 4 by $\Lambda(\Delta)$.

Lyapunov-like functions used to discuss stability for differential equations require an appropriate definition of the derivative of the Lyapunov function $V(t, x)$ along the studied differential equations. For fractional differential equations, some authors (see, for example, [14]) defined and used the so-called Caputo fractional derivative of Lyapunov function ${ }_{t_{0}}^{c} D^{q} V(t, x(t))$ where $x(t)$ is the unknown solution of the studied fractional differential equation. This approach requires the function to be smooth enough (at least continuously differentiable), and also some conditions involved are quite restrictive. Other authors used the so-called Dini fractional derivative of Lyapunov function $[17,18]$. This is based on the 
Dini derivative of the Lyapunov function $V(t, x)$ among the ordinary differential equation $x^{\prime}=f(t, x)$ given by

$$
D_{+} V(t, x)=\limsup _{h \rightarrow 0} \frac{1}{h+}[V(t, x)-V(t-h, x-h f(t, x))] .
$$

The authors generalized (9) to the fractional Dini derivative along FrDE (4) by

$$
{ }^{c} D_{+}^{q} V(t, x)=\limsup _{h \rightarrow 0+} \frac{1}{h^{q}}\left[V(t, x)-V\left(t-h, x-h^{q} f(t, x)\right)\right] .
$$

This definition requires only the continuity of the Lyapunov function. However, it can be quite restrictive (see Example 5), and it can present some problems (see Example 6).

In this paper we introduce the derivative of the Lyapunov function based on the Caputo fractional Dini derivative of a function $m(t)$ given by (3). We define the generalized $\mathrm{Ca}$ puto fractional Dini derivative of the function $V(t, x) \in \Lambda\left(\left[t_{0}, T\right), \Delta\right)$ along trajectories of solutions of the system $\operatorname{FrDE}(4)$ as follows:

$$
\begin{aligned}
{ }_{(4)}^{c} D_{+}^{q} V\left(t, x ; t_{0}, x_{0}\right) \\
=\limsup _{h \rightarrow 0^{+}} \frac{1}{h^{q}}\left\{V(t, x)-V\left(t_{0}, x_{0}\right)\right. \\
\left.\quad-\sum_{r=1}^{\left[\frac{t-t_{0}}{h}\right]}(-1)^{r+1} q \operatorname{Cr}\left[V\left(t-r h, x-h^{q} f(t, x)\right)-V\left(t_{0}, x_{0}\right)\right]\right\} \quad \text { for } t \geq t_{0},
\end{aligned}
$$

where $t \in\left(t_{0}, T\right), x, x_{0} \in \Delta$, and there exists $h_{1}>0$ such that $t-h \in\left[t_{0}, T\right), x-h^{q} f(t, x) \in \Delta$ for $0<h \leq h_{1}$.

Using the relation $\limsup _{h \rightarrow 0^{+}} \frac{1}{h^{q}} \sum_{r=0}^{\left[\frac{t-t_{0}}{h}\right]}(-1)^{r} q C r=D_{0}^{q}(1)=\frac{\left(t-t_{0}\right)^{-q}}{\Gamma(1-q)}$, formula (11) could be reduced to

$$
\begin{aligned}
& { }_{(4)}^{c} D_{+}^{q} V\left(t, x ; t_{0}, x_{0}\right) \\
& =\limsup _{h \rightarrow 0^{+}} \frac{1}{h^{q}}\left\{V(t, x)-\sum_{r=1}^{\left[\frac{t-t_{0}}{h}\right]}(-1)^{r+1} q C r V\left(t-r h, x-h^{q} f(t, x)\right)\right\} \\
& \quad-V\left(t_{0}, x_{0}\right) \frac{\left(t-t_{0}\right)^{-q}}{\Gamma(1-q)} \text { for } t \geq t_{0} .
\end{aligned}
$$

Proposition 2 Let the function $V \in \Lambda(\Delta)$, i.e., $V(t, x)=V(x)$. Then

$$
{ }_{(4)}^{c} D_{+}^{q} V\left(t, x ; t_{0}, x_{0}\right)={ }^{c} D_{+}^{q} V(x)+\left(V(x)-V\left(x_{0}\right)\right) \frac{\left(t-t_{0}\right)^{-q}}{\Gamma(1-q)} .
$$

Proof In the case of the Lyapunov function $V(t, x)=V(x)$, formula (12) reduces to

$$
\begin{aligned}
{ }_{(4)}^{c} & D_{+}^{q} V\left(t, x ; t_{0}, x_{0}\right) \\
\quad & \limsup _{h \rightarrow 0^{+}} \frac{1}{h^{q}}\left(V(x)-V\left(x-h^{q} f(t, x)\right)\right)+\left(V(x)-V\left(x_{0}\right)\right) \frac{\left(t-t_{0}\right)^{-q}}{\Gamma(1-q)} .
\end{aligned}
$$




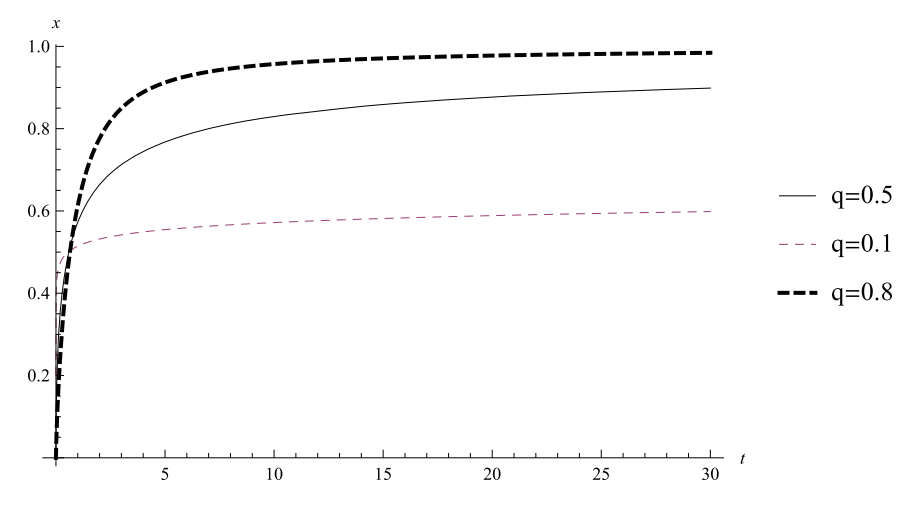

Figure 9 Graphs of solutions of ${ }_{0}^{c} D^{q} x+x(t)=1$ for various $q$.

Example 5 Let $V \in \Lambda\left(\mathbb{R}_{+}, \mathbb{R}\right)$ be such that $V(t, x)=m^{2}(t) x^{2}$, where $m \in C^{1}\left(\mathbb{R}_{+}, \mathbb{R}\right)$ and $x \in \mathbb{R}$.

First we apply formula (10) to obtain the fractional Dini derivative of the considered Lyapunov function. We obtain

$$
\begin{aligned}
{ }^{c} D_{+}^{q} V(t, x)= & \lim _{h \rightarrow 0^{+}} \sup \frac{1}{h^{q}}\left[m^{2}(t) x^{2}-m^{2}(t-h)\left(x-h^{q} f(t, x)\right)^{2}\right] \\
= & \lim _{h \rightarrow 0^{+}} \sup \left[\left(\frac{m(t)-m(t-h)}{h} x h^{1-q}+m(t-h) f(t, x)\right)\right. \\
& \left.\times\left((m(t)+m(t-h)) x-m(t-h) h^{q} f(t, x)\right)\right] \\
= & 2 x m^{2}(t) f(t, x) \quad \text { for } t \geq t_{0} .
\end{aligned}
$$

Note that the fractional Dini derivative ${ }^{c} D_{+}^{q} V(t, x)$ does not depend on the order $q$ of the fractional differential equation. However, as shown in Example 1, the behavior of the fractional derivative depends significantly on the fractional order $q$. The same can be said about the solutions of fractional differential equations. For example, let us consider the simple Caputo fractional differential equation ${ }_{0}^{c} D^{q} x+x(t)=1, x(0)=0$ whose solution is given by $x(t)=t^{q} E_{q, 1+q}\left(-t^{q}\right)$. From Figure 9 it can be seen that $\lim _{t \rightarrow \infty} x(t)=a$, where $a$ is different for different values of the order $q$ of the fractional differential equation.

Next we use (12) to obtain the Caputo fractional Dini derivative of $V(t, x)$. Let $t \geq t_{0}$. Then

$$
\begin{aligned}
{ }_{(4)}^{c} D_{+}^{q} V\left(t, x ; t_{0}, x_{0}\right) \\
=\lim _{h \rightarrow 0^{+}} \sup \frac{1}{h^{q}}\left[m^{2}(t) x^{2}-\sum_{r=1}^{\left[\frac{t-t_{0}}{h}\right]}(-1)^{r+1} q C r m^{2}(t-r h)\left(x-h^{q} f(t, x)\right)^{2}\right] \\
\quad-m^{2}\left(t_{0}\right) x_{0}^{2} \frac{\left(t-t_{0}\right)^{-q}}{\Gamma(1-q)} \\
=x^{2} \lim _{h \rightarrow 0^{+}} \sup \frac{1}{h^{q}} \sum_{r=0}^{\left[\frac{t-t_{0}}{h}\right]}(-1)^{r} q \operatorname{Crm}^{2}(t-r h)-m^{2}\left(t_{0}\right) x_{0}^{2} \frac{\left(t-t_{0}\right)^{-q}}{\Gamma(1-q)}
\end{aligned}
$$




$$
\begin{aligned}
& -2 x f(t, x) \lim _{h \rightarrow 0^{+}} \sup \sum_{r=1}^{\left[\frac{t-t_{0}}{h}\right]}(-1)^{r} q C r m^{2}(t-r h) \\
& +(f(t, x))^{2} \lim _{h \rightarrow 0^{+}} \sup h^{q} \sum_{r=1}^{\left[\frac{t-t_{0}}{h}\right]}(-1)^{r} q C r m^{2}(t-r h) .
\end{aligned}
$$

Now using (2), the relation between G-L and R-L fractional derivatives

$$
{ }_{t_{0}}^{G L} D_{+}^{q}\left[m^{2}(t)\right]=\lim _{h \rightarrow 0^{+}} \sup \frac{1}{h^{q}} \sum_{r=0}^{\left[\frac{t-t_{0}}{h}\right]}(-1)^{r} q C r m^{2}(t-r h)={ }_{t_{0}}^{R L} D^{q}\left(m^{2}(t)\right),
$$

we obtain from (15) the following formula for the Caputo fractional Dini derivative:

$$
\begin{aligned}
{ }_{(4)}^{c} & D_{+}^{q} V\left(t, x ; t_{0}, x_{0}\right) \\
& =2 x m^{2}(t) f(t, x)+x_{t_{0}}^{2 R L} D^{q}\left(m^{2}(t)\right)-\left(x_{0}\right)^{2} m^{2}\left(t_{0}\right) \frac{\left(t-t_{0}\right)^{-q}}{\Gamma(1-q)} \quad \text { for } t \geq t_{0},
\end{aligned}
$$

or

$$
\begin{aligned}
& { }_{(4)}^{c} D_{+}^{q} V\left(t, x ; t_{0}, x_{0}\right) \\
& \quad=2 x m^{2}(t) f(t, x)+x_{t_{0}}^{2 C} D^{q}\left(m^{2}(t)\right)+\left(x^{2}-x_{0}^{2}\right) m^{2}\left(t_{0}\right) \frac{\left(t-t_{0}\right)^{-q}}{\Gamma(1-q)} \quad \text { for } t \geq t_{0} .
\end{aligned}
$$

Note that the Caputo fractional Dini derivative ${ }_{(4)}^{c} D_{+}^{q} V\left(t, x ; t_{0}, x_{0}\right)$ depends significantly not only on the order $q$ of the fractional differential equation but also on the initial data.

The Dini derivative of the Lyapunov function $(q=1)$ given by $(9)$ is

$$
D_{+} V(t, x)=2 x m^{2}(t) f(t, x)+x^{2} \frac{d}{d t}\left[m^{2}(t)\right], \quad t \geq t_{0} .
$$

Let $m(t) \equiv 1$ (i.e., we consider the quadratic Lyapunov function $V(x)=x^{2}$ ). Note that for $q \rightarrow 1$ the limit ${ }_{(4)}^{c} D_{+}^{q} V\left(t, x ; t_{0}, x_{0}\right)=2 x f(t, x)$ in (17) coincides with the corresponding derivative $D_{+} V(x)=2 x f(t, x)$ in the ordinary case (18).

Let $m(t) \not \equiv 1$. For $q \rightarrow 1$, the limit ${ }_{(4)}^{c} D_{+}^{q} V\left(t, x ; t_{0}, x_{0}\right)=2 x m^{2}(t) f(t, x)+x_{t_{0}}^{2 C} D^{q}\left(m^{2}(t)\right)$ in (17) is similar to $D_{+} V(t, x)$ in the ordinary case (18) where the ordinary derivative of $m^{2}(t)$ is replaced by the fractional one.

The Caputo fractional Dini derivative given by formula (11) seems to be the natural generalization of the Dini derivative (9) for ordinary differential equations.

\section{Fractional differential inequalities and comparison results for the scalar FrDE}

Again in this section we assume $0<q<1$. Now we will give some comparison results. Note that similar results were obtained by the authors in paper [19].

Lemma 1 ([19]) Let $m \in C\left[\left[t_{0}, T\right], \mathbb{R}\right]$ and suppose that there exists $t^{*} \in\left(t_{0}, T\right]$ such that $m\left(t^{*}\right)=0$ and $m(t)<0$ for $t_{0} \leq t<t^{*}$. Then, if the Caputo fractional Dini derivative (3) of $m$ exists at $t^{*}$, then the inequality ${ }_{t_{0}}^{c} D_{+}^{q} m\left(t^{*}\right)>0$ holds. 
We will use the following comparison result, which generalizes the result in [19].

Lemma 2 (Comparison result by Caputo fractional Dini derivative) Assume that the following conditions are satisfied:

(1) The function $x^{*}(t)=x\left(t ; t_{0}, x_{0}\right), x^{*} \in C^{q}\left(\left[t_{0}, T\right], \Delta\right)$, is a solution of FrDE (4), where

$\Delta \subset \mathbb{R}^{n}, 0 \in \Delta, t_{0}, T \in \mathbb{R}_{+}: t_{0}<T$ are given constants, $x_{0} \in \Delta$.

(2) The function $g \in C\left[\left[t_{0}, T\right] \times \mathbb{R}, \mathbb{R}\right]$ and satisfies condition (H1).

(3) The function $V \in \Lambda\left(\left[t_{0}, T\right], \Delta\right)$ and, for any $t \in\left[t_{0}, T\right]$, the inequality

$$
{ }_{(4)}^{c} D_{+}^{q} V\left(t, x^{*}(t) ; t_{0}, x_{0}\right) \leq(\geq) g\left(t, V\left(t, x^{*}(t)\right)\right)
$$

holds.

(4) The function $u^{*}(t)=u\left(t ; t_{0}, u_{0}\right), u^{*} \in C^{q}\left(\left[t_{0}, T\right], \mathbb{R}\right)$, is the maximal solution (minimal solution) of the initial value problem (5).

Then the inequality $V\left(t_{0}, x_{0}\right) \leq(\geq) u_{0}$ implies $V\left(t, x^{*}(t)\right) \leq(\geq) u^{*}(t)$ for $t \in\left[t_{0}, T\right]$.

Proof Case 1. Suppose that all inequalities are $\leq$. This case is proved in [19].

Case 2. Suppose that all inequalities are $\geq$. Let the function $u^{*}(t)=u\left(t ; t_{0}, u_{0}\right), u^{*} \in$ $C^{q}\left(\left[t_{0}, T\right], \mathbb{R}\right)$, be the minimal solution of the initial value problem (5) such that $V\left(t_{0}, x_{0}\right) \geq$ $u_{0}$.

Let $\eta \in\left(0, L_{t_{0}}\right]$ be an arbitrary number where the number $L_{t_{0}}>0$ exists according to condition (2) of Lemma 2. Consider the initial value problem for the scalar FrDE

$$
{ }_{t_{0}}^{c} D^{q} u=g(t, u)-\eta \quad \text { for } t \in\left[t_{0}, T\right], \quad u\left(t_{0}\right)=u_{0}-\eta .
$$

The function $u(t, \eta)$ is a solution of the scalar fractional differential equation (19) iff it satisfies the Volterra fractional integral equation (Lemma 6.2 [23])

$$
u(t, \eta)=u_{0}-\eta+\frac{1}{\Gamma(q)} \int_{t_{0}}^{t}(t-s)^{q-1}(g(s, u(s, \eta))-\eta) d s \quad \text { for } t \in\left[t_{0}, T\right] .
$$

Let the function $m(t) \in C\left[\left[t_{0}, T\right], \mathbb{R}_{-}\right]$be $m(t)=-V\left(t, x^{*}(t)\right)$. We now prove that

$$
m(t)<-u(t, \eta) \text { for } t \in\left[t_{0}, T\right] .
$$

Note that inequality (21) holds for $t=t_{0}$ since $m\left(t_{0}\right)=-V\left(t_{0}, x_{0}\right) \leq-u_{0}<-u_{0}+\eta=$ $-u\left(t_{0}, \eta\right)$. Assume that inequality (21) is not true. Then there exists a point $t^{*}$ such that $m\left(t^{*}\right)=-u\left(t^{*}, \eta\right), m(t)<-u(t, \eta)$ for $t \in\left[t_{0}, t^{*}\right)$. Now Lemma 1 (applied to $m(t)+u(t, \eta)$ ) yields ${ }^{c} D_{+}^{q}\left(m\left(t^{*}\right)+u\left(t^{*}, \eta\right)\right)>0$, i.e.,

$$
\begin{aligned}
{ }^{c} D_{+}^{q} m\left(t^{*}\right) & >-{ }^{c} D_{+}^{q}\left(u\left(t^{*}, \eta\right)\right)=-{ }^{c} D^{q} u\left(t^{*}, \eta\right)=-g\left(t^{*}, u\left(t^{*}, \eta\right)\right)+\eta \\
& >-g\left(t^{*},-m\left(t^{*}\right)\right) .
\end{aligned}
$$

From condition (1) of Lemma 2 the function $x^{*}(t)$ satisfies the following initial value problem for the system FrDE:

$$
{ }_{t_{0}}^{c} D_{+}^{q} x=f(t, x), \quad x\left(t_{0}\right)=x_{0}, \quad t \in\left[t_{0}, T\right] .
$$


Then, for $t \in\left(t_{0}, T\right]$, the equality

$$
\limsup _{h \rightarrow 0+} \frac{1}{h^{q}}\left[x^{*}(t)-x_{0}-S\left(x^{*}(t), h\right)\right]=f\left(t, x^{*}(t)\right)
$$

holds, where

$$
S\left(x^{*}(t), h\right)=\sum_{r=1}^{\left[\frac{t-t_{0}}{h}\right]}(-1)^{r+1} q C r\left[x^{*}(t-r h)-x_{0}\right] .
$$

Therefore,

$$
S\left(x^{*}(t), h\right)=x^{*}(t)-x_{0}-h^{q} f\left(t, x^{*}(t)\right)-\Xi\left(h^{q}\right)
$$

or

$$
x^{*}(t)-h^{q} f\left(t, x^{*}(t)\right)=S\left(x^{*}(t), h\right)+x_{0}+\Xi\left(h^{q}\right)
$$

with $\frac{\Xi\left(h^{q}\right)}{h^{q}} \rightarrow 0$ as $h \rightarrow 0$. Then, for any $t \in\left(t_{0}, T\right]$, we obtain

$$
\begin{aligned}
m(t) & -m\left(t_{0}\right)-\sum_{r=1}^{\left[\frac{t-t_{0}}{h}\right]}(-1)^{r+1} q C r\left[m(t-r h)-m\left(t_{0}\right)\right] \\
= & -\left\{V\left(t, x^{*}(t)\right)-V\left(t_{0}, x_{0}\right)\right. \\
& \left.-\sum_{r=1}^{\left[\frac{t-t_{0}}{h}\right]}(-1)^{r+1} q C r\left[V\left(t-r h, x^{*}(t)-h^{q} f\left(t, x^{*}(t)\right)\right)-V\left(t_{0}, x_{0}\right)\right]\right\} \\
& \left.+\sum_{r=1}^{t-t_{0}}\right] \\
- & {\left[V ( t - r ) ^ { r } q C r \left\{\left[V\left(t-r h, S\left(x^{*}(t), h\right)+x_{0}+\Xi\left(h^{q}\right)\right)-V\left(t_{0}, x_{0}\right)\right]\right.\right.}
\end{aligned}
$$

Since $V$ is locally Lipschitzian in its second argument with a Lipschitz constant $L>0$, we obtain

$$
\begin{aligned}
& \sum_{r=1}^{\left[\frac{t-t_{0}}{h}\right]}(-1)^{r} q C r\left\{V\left(t-r h, S\left(x^{*}(t), h\right)+x_{0}+\Xi\left(h^{q}\right)\right)\right. \\
& \left.\quad-V\left(t-r h, x^{*}(t-r h)\right)\right\} \\
& \leq L\left\|\sum_{r=1}^{\left[\frac{t-t_{0}}{h}\right]} q C r\left(S\left(x^{*}(t), h\right)+\Xi\left(h^{q}\right)-\left(x^{*}(t-r h)-x_{0}\right)\right)\right\| \\
& \leq L\left\|\sum_{r=1}^{\left[\frac{t-t_{0}}{h}\right]}(-1)^{r} q C r \sum_{j=1}^{\left[\frac{t-t_{0}}{h}\right]} q C j\left(x^{*}(t-j h)-x_{0}\right)-\sum_{r=1}^{\left[\frac{t-t_{0}}{h}\right]} q C r\left(\left(x^{*}(t-r h)-x_{0}\right)\right)\right\|
\end{aligned}
$$




$$
\begin{aligned}
& +L \Xi\left(h^{q}\right) \sum_{r=1}^{\left[\frac{t-t_{0}}{h}\right]} q C r \\
= & L\left\|\left(\sum_{r=0}^{\left[\frac{t-t_{0}}{h}\right]}(-1)^{r} q C r\right)\left(\sum_{j=1}^{\left[\frac{t-t_{0}}{h}\right]} q C j\left(x^{*}(t-j h)-x_{0}\right)\right)\right\|+L \Xi\left(h^{q}\right) \sum_{r=1}^{\left[\frac{t-t_{0}}{h}\right]} q C r .
\end{aligned}
$$

Substitute (26) in (25), divide both sides by $h^{q}$, take the limit as $h \rightarrow 0^{+}$, use $\sum_{r=0}^{\infty} q C r z^{r}=$ $(1+z)^{q}$ if $|z| \leq 1$, and we obtain for any $t \in\left(t_{0}, T\right]$ the inequality (note (3) and (12) and condition (3) of Lemma 2)

$$
\begin{aligned}
{ }^{c} D_{+}^{q} m(t) \leq & -{ }_{(4)}^{c} D_{+}^{q} V\left(t, x^{*}(t) ; t_{0}, x_{0}\right)+L \lim _{h \rightarrow 0+} \frac{\Lambda\left(h^{q}\right)}{h^{q}} \lim _{h \rightarrow 0+} \sum_{r=1}^{\left[\frac{t-t_{0}}{h}\right]} q C r \\
& +L \lim _{h \rightarrow 0^{+}} \sup \left\|\left(\sum_{r=0}^{\left[\frac{t-t_{0}}{h}\right]}(-1)^{r} q C r\right)\left(\frac{1}{h^{q}} \sum_{j=1}^{\left[\frac{t-t_{0}}{h}\right]} q C j\left(x^{*}(t-j h)-x_{0}\right)\right)\right\| \\
= & -{ }_{(4)}^{c} D_{+}^{q} V\left(t, x^{*}(t) ; t_{0}, x_{0}\right) \leq-g\left(t, V\left(t, x^{*}(t)\right)\right)=-g(t,-m(t)) .
\end{aligned}
$$

Now (27) with $t=t^{*}$ contradicts (22). Therefore (21) holds.

We now show if $\eta_{2}<\eta_{1}$ then

$$
u\left(t, \eta_{2}\right)>u\left(t, \eta_{1}\right) \quad \text { for } t \in\left[t_{0}, T\right] .
$$

Note that inequality (28) holds for $t=t_{0}$. Assume that inequality (28) is not true. Then there exists a point $t^{*}$ such that $u\left(t^{*}, \eta_{2}\right)=u\left(t^{*}, \eta_{1}\right)$ and $u\left(t, \eta_{2}\right)>u\left(t, \eta_{1}\right)$ for $t \in\left[t_{0}, t^{*}\right)$. Now Lemma 1 (applied to $u\left(t, \eta_{1}\right)-u\left(t, \eta_{2}\right)$ ) yields ${ }_{t_{0}}^{c} D_{+}^{q}\left(u\left(t^{*}, \eta_{1}\right)-u\left(t^{*}, \eta_{2}\right)\right)>0$. However,

$$
{ }_{t_{0}}^{c} D_{+}^{q}\left(u\left(t^{*}, \eta_{1}\right)-u\left(t^{*}, \eta_{2}\right)\right)=g\left(t^{*}, u\left(t^{*}, \eta_{1}\right)\right)-\eta_{1}-\left[g\left(t^{*}, u\left(t^{*}, \eta_{2}\right)\right)-\eta_{2}\right]=\eta_{2}-\eta_{1}<0,
$$

a contradiction. Thus (28) is true.

Recall $\eta \in\left(0, L_{t_{0}}\right]$. Now (21) and (28) guarantee that the family of solutions $\{u(t, \eta)\}$, $t \in\left[t_{0}, T\right]$ of (19) is uniformly bounded, i.e., there exists $K>0$ with $|u(t, \eta)| \leq K$ for $(t, \eta) \in\left[t_{0}, T\right] \times\left[0, L_{t_{0}}\right]$. Let $M=\sup \left\{|g(t, x)|:(t, x) \in\left[t_{0}, T\right] \times[-K, K]\right\}$. Take a decreasing sequence of positive numbers $\left\{\eta_{j}\right\}_{j=0}^{\infty}, \eta_{0} \leq L_{t_{0}}$, such that $\lim _{j \rightarrow \infty} \eta_{j}=0$ and consider the sequence of functions $u\left(t ; \eta_{j}\right)$. Now, for $t_{1}, t_{2} \in\left[t_{0}, T\right], t_{1}<t_{2}$, we have

$$
\begin{aligned}
& \left|u\left(t_{2}, \eta_{j}\right)-u\left(t_{1}, \eta_{j}\right)\right| \\
& \leq \frac{1}{\Gamma(q)} \mid \int_{t_{0}}^{t_{1}}\left(\left(t_{2}-s\right)^{q-1}-\left(t_{1}-s\right)^{q-1}\right)\left(g\left(s, u\left(s, \eta_{j}\right)\right)-\eta_{j}\right) d s \\
& \quad-\int_{t_{1}}^{t_{2}}\left(\left(t_{2}-s\right)^{q-1}\right)\left(g\left(s, u\left(s, \eta_{j}\right)\right)-\eta_{j}\right) d s \mid \leq 2 \frac{[M+1]}{q \Gamma(q)}\left(t_{2}-t_{1}\right)^{q} .
\end{aligned}
$$

Thus the family $\left\{u\left(t ; \eta_{j}\right)\right\}$ is equicontinuous on $\left[t_{0}, T\right]$. The Arzela-Ascoli theorem guarantees that there exists a subsequence, $\left\{u\left(t ; \eta_{j_{k}}\right)\right\}$ and $w \in C\left[t_{0}, T\right]$ with $u\left(t ; \eta_{j_{k}}\right) \rightarrow w$ in $C\left[t_{0}, T\right]$ as $k \rightarrow \infty$. Take the limit in (20) as $k \rightarrow \infty$, and we see that $w(t)$ satisfies the 
initial value problem (5) for $t \in\left[t_{0}, T\right]$. Now from (21) we have $m(t) \leq-w(t) \leq-u^{*}(t)$ on $\left[t_{0}, T\right]$ or $-V\left(t, x^{*}(t)\right) \leq-u^{*}(t)$.

If $g(t, x) \equiv 0$ in Lemma 2, we obtain the following result.

Corollary 1 Assume that the following conditions are satisfied:

(1) The function $x^{*}(t)=x\left(t ; t_{0}, x_{0}\right), x^{*} \in C^{q}\left(\left[t_{0}, T\right], \Delta\right)$, is a solution of FrDE (4) where $\Delta \subset \mathbb{R}^{n}, 0 \in \Delta$.

(2) The function $V \in \Lambda\left(\left[t_{0}, T\right], \Delta\right)$ and for $t \in\left[t_{0}, T\right]$ the inequality

$$
{ }_{(4)}^{c} D_{+}^{q} V\left(t, x^{*}(t) ; t_{0}, x_{0}\right) \leq(\geq) 0
$$

holds.

Then, for $t \in\left[t_{0}, T\right]$, the inequality $V\left(t, x^{*}(t)\right) \leq(\geq) V\left(t_{0}, x_{0}\right)$ holds.

Note that a similar result to Corollary 1 for Dini fractional derivatives is proved in [18] (Corollary 2.2 considers the case when all inequalities are $\leq$ ). We note that (10) could lead to some problems.

Example 6 Let $V: \mathbb{R}_{+} \times \mathbb{R} \rightarrow \mathbb{R}_{+}$be given by $V(t, x)=\sin ^{2} t x^{2}$ and $t_{0}=0$. It is locally Lipschitz with respect to its second argument $x$.

First, apply formula (14) to obtain the derivative of $V$, namely

$$
{ }^{c} D_{+}^{q} V(t, x)=2 x \sin ^{2}(t) f(t, x) .
$$

From (16) we obtain the Caputo fractional Dini derivative of $V$, namely

$$
{ }_{(4)}^{c} D_{+}^{q} V\left(t, x ; 0, x_{0}\right)=2 x \sin ^{2}(t) f(t, x)+x_{0}^{2 R L} D^{q}(\sin (t))^{2} .
$$

Use $(\sin (t))^{2}=0.5-0.5 \cos (2 t)$ and ${ }_{0}^{R L} D^{q} \cos (2 t)=2^{q} \cos \left(2 t+\frac{q \pi}{2}\right)$ and obtain

$$
{ }_{(4)}^{c} D_{+}^{q} V\left(t, x ; 0, x_{0}\right)=2 x \sin ^{2}(t) f(t, x)+x^{2}\left(0.5 \frac{t^{-q}}{\Gamma(1-q)}+2^{q-1} \cos \left(2 t+\frac{q \pi}{2}\right)\right) .
$$

Let $f(t, x) \equiv 0$. The solution of (4) for $n=1$ and $t_{0}=0$ is $x(t) \equiv x_{0}, t \geq 0$ and $V(t, x(t))=$ $x_{0}^{2} \sin ^{2} t$. Note that ${ }^{c} D_{+}^{q} V(t, x)=0$ and all the conditions of Corollary 2.2 [18] are satisfied, so the inequality $V(t, x(t)) \leq V\left(t_{0}, x_{0}\right), t \geq t_{0}$, has to hold. However, the inequality $x_{0}^{2} \sin ^{2} t \leq$ $x_{0}^{2} \sin ^{2} 0=0$ is not satisfied for all $t \geq t_{0}$.

From (31) we obtain for the Caputo fractional Dini derivative

$$
{ }_{(4)}^{c} D_{+}^{q} V\left(t, x ; 0, x_{0}\right)=x^{2}\left(0.5 \frac{t^{-q}}{\Gamma(1-q)}+2^{q-1} \cos \left(2 t+\frac{q \pi}{2}\right)\right) \equiv x^{2} p(t),
$$

where $p(t)=0.5 \frac{t^{-q}}{\Gamma(1-q)}+2^{q-1} \cos \left(2 t+\frac{q \pi}{2}\right)$. The sign of the Caputo fractional Dini derivative of $V(t, x)$ changes (see Figure 10 for the graph of $p(t), q=0.2,0.5,0.8)$. Therefore, the conditions of Corollary 1 are not satisfied.

Now let $V: \mathbb{R}_{+} \times \mathbb{R} \rightarrow \mathbb{R}_{+}$be given by $V(t, x)=x^{2}$. According to (16) for $m(t) \equiv 1$, we get ${ }_{(4)}^{c} D_{+}^{q} V\left(t, x ; 0, x_{0}\right)=\frac{x^{2}-x_{0}^{2}}{t^{q} \Gamma(1-q)}+2 x f(t, x)$. Let $f(t, x)=-\frac{x}{t^{q} \Gamma(1-q)}$. Then ${ }_{(4)}^{c} D_{+}^{q} V\left(t, x ; 0, x_{0}\right) \leq 0$, 


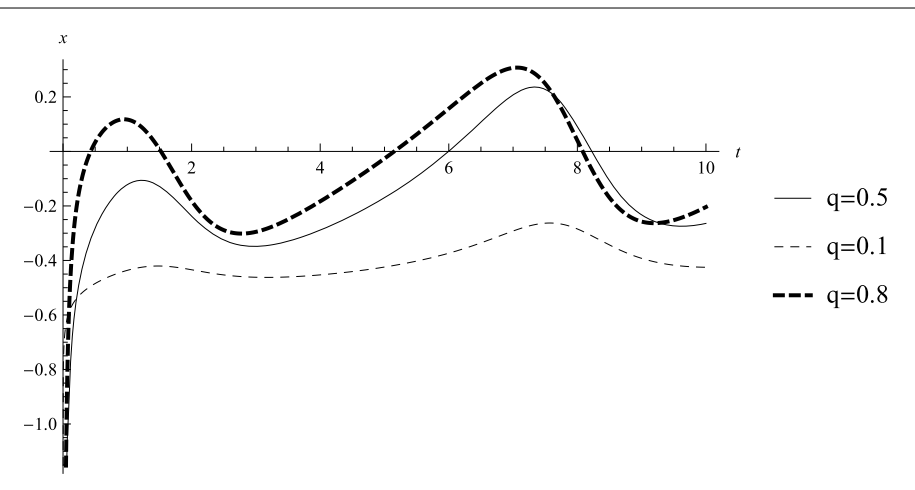

Figure 10 Example 6: $q=0.2,0.5$ and 0.8 .

and according to Corollary 1 the inequality $\left|x\left(t ; 0, x_{0}\right)\right| \leq\left|x_{0}\right|, t \geq 0$, holds for any solution $x\left(t ; 0, x_{0}\right)$ of $(4)$.

The result of Lemma 2 is also true on the half line (see [19] for the case when all inequalities are $\leq$ ).

Corollary 2 Assume that the following conditions are satisfied:

(1) The function $x^{*}(t)=x\left(t ; t_{0}, x_{0}\right), x^{*} \in C^{q}\left(\left[t_{0}, \infty\right), \Delta\right)$, is a solution of FrDE (4) where $\Delta \subset \mathbb{R}^{n}, 0 \in \Delta$.

(2) The function $g \in C\left[\left[t_{0}, \infty\right) \times \mathbb{R}, \mathbb{R}\right]$ and satisfies condition (H1).

(3) The function $V \in \Lambda\left(\left[t_{0}, \infty\right), \Delta\right)$, and for any points $t \geq t_{0}$ and $x \in \Delta$ the inequality

$$
{ }_{(4)}^{c} D_{+}^{q} V\left(t, x^{*}(t) ; t_{0}, x_{0}\right) \leq(\geq) g\left(t, V\left(t, x^{*}(t)\right)\right)
$$

holds.

(4) The function $u^{*}(t)=u\left(t ; t_{0}, u_{0}\right), u^{*} \in C^{q}\left(\left[t_{0}, \infty\right), \mathbb{R}\right)$ is the maximal solution (minimal solution) of the initial value problem (5).

Then the inequality $V\left(t_{0}, x_{0}\right) \leq(\geq) u_{0}$ implies $V\left(t, x^{*}(t)\right) \leq(\geq) u^{*}(t)$ for $t \geq t_{0}$.

\section{Main results}

We obtain sufficient conditions for strict stability of the system $\operatorname{FrDE}(4)$. Again we assume $0<q<1$.

Theorem 1 Let the following conditions be satisfied:

(1) The functions $g_{i} \in C\left[\mathbb{R}_{+} \times \mathbb{R}, \mathbb{R}\right], g_{i}(t, 0) \equiv 0, i=1,2$, and satisfy condition (H1).

(2) There exists a function $V_{1} \in \Lambda\left(\mathbb{R}_{+}, \mathbb{R}^{n}\right)$ such that $V_{1}(t, 0) \equiv 0$ for $t \in \mathbb{R}_{+}$and

(i) the inequality

$$
{ }_{(4)}^{c} D_{+}^{q} V_{1}\left(t, x ; t_{0}, x_{0}\right) \leq g_{1}\left(t, V_{1}(t, x)\right)
$$

holds for any $t_{0}, t \in \mathbb{R}_{+}, t \geq t_{0}$ and $x, x_{0} \in \mathbb{R}^{n}$;

(ii) $a(\|x\|) \leq V_{1}(t, x)$ for $t \in \mathbb{R}_{+}, x \in \mathbb{R}^{n}$, where $a \in \mathcal{K}$.

(3) There exists a function $V_{2} \in \Lambda\left(\mathbb{R}_{+}, \mathbb{R}^{n}\right)$ such that 
(iii) the inequality

$$
{ }_{(4)}^{c} D_{+}^{q} V_{2}\left(t, x ; t_{0}, x_{0}\right) \geq g_{2}\left(t, V_{2}(t, x)\right)
$$

holds for any $t_{0}, t \in \mathbb{R}_{+}, t \geq t_{0}$ and $x, x_{0} \in \mathbb{R}^{n}$;

(iv) $c(\|x\|) \leq V_{2}(t, x) \leq b(\|x\|)$ for $t \in \mathbb{R}_{+}, x \in \mathbb{R}^{n}$, where $b, c \in \mathcal{K}$.

(4) The zero solution of FrDE (6) is strictly stable in couple.

Then the zero solution of the system FrDE (4) is strictly stable.

Proof Let $\varepsilon_{1}>0$ be an arbitrary number. From condition (4) there exists $\delta_{1}=\delta_{1}\left(t_{0}, \varepsilon\right) \geq 0$, and for any $\delta_{2} \in\left(0, \delta_{1}\right]$ there exists $\varepsilon_{2} \in\left(0, \delta_{2}\right]$ such that $\left|u_{0}\right|<\delta_{1}$ and $\left|v_{0}\right|>\delta_{2}$ imply

$$
\begin{aligned}
& \left|u\left(t ; t_{0}, u_{0}\right)\right|<a(\varepsilon) \quad \text { for } t \geq t_{0}, \\
& \left|v\left(t ; t_{0}, v_{0}\right)\right|>\varepsilon_{2} \quad \text { for } t \geq t_{0}
\end{aligned}
$$

where the couple $\left(u\left(t ; t_{0}, u_{0}\right), v\left(t ; t_{0}, v_{0}\right)\right)$ is a solution of $(6)$.

Since $V_{1}\left(t_{0}, 0\right)=0$, there exists $\delta_{3}=\delta_{3}\left(t_{0}, \varepsilon\right), \delta_{3} \in\left(0, \delta_{1}\right)$ such that $V_{1}\left(t_{0}, x\right)<\delta_{1}$ for $\|x\|<\delta_{3}$. Let $\delta_{4} \in\left(0, \delta_{3}\right]$ be an arbitrary number. Then there exists $\delta_{5} \in\left(0, \delta_{1}\right]$ such that $c\left(\delta_{4}\right)>\delta_{5}$. According to the above, for $\delta_{5}$ there exists $\varepsilon_{2} \in\left(0, \delta_{5}\right]$ such that $\left|v_{0}\right|>\delta_{5}$ implies (33). Choose $\varepsilon_{3} \in\left(0, \delta_{4}\right]$ such that $b\left(\varepsilon_{3}\right)<\varepsilon_{2}$. Choose $x_{0} \in \mathbb{R}^{n}$ with $\delta_{4}<\left\|x_{0}\right\|<\delta_{3}$. Let $x^{*}(t)=x\left(t ; t_{0}, x_{0}\right)$ be a solution of the IVP for FrDE (4) for the initial data $\left(t_{0}, x_{0}\right)$.

Let $u_{0}=V_{1}\left(t_{0}, x_{0}\right)$ and $v_{0}=V_{2}\left(t_{0}, x_{0}\right)$. Let the couple $\left(u\left(t ; t_{0}, u_{0}\right), v\left(t ; t_{0}, v_{0}\right)\right)$ be the solution of $\operatorname{FrDE}(6)$ for the initial values $\left(u_{0}, v_{0}\right)$ such that the components are maximal and minimal solutions of the first and second equation, respectively. From the choice of $x_{0}$ it follows that $\left|u_{0}\right|<\delta_{1}$. Therefore, the component $u\left(t ; t_{0}, u_{0}\right)$ satisfies inequality (32). From the choice of $x_{0}$ and condition (3)(iv) it follows that $\left|v_{0}\right|=V_{2}\left(t_{0}, x_{0}\right) \geq c\left(\left\|x_{0}\right\|\right)>c\left(\delta_{4}\right)>\delta_{5}$. Therefore, the component $v\left(t ; t_{0}, v_{0}\right)$ satisfies inequality (33).

According to Corollary 2 applied to the solution $x^{*}(t)$ and condition (2)(ii), we obtain $a\left(\left\|x^{*}(t)\right\|\right) \leq V\left(t, x^{*}(t)\right) \leq u\left(t ; t_{0}, u_{0}\right)<a\left(\varepsilon_{1}\right), t \geq t_{0}$. Therefore $\left\|x^{*}(t)\right\|<\varepsilon_{1}$ for $t \geq t_{0}$.

According to Corollary 2 applied to the solution $x^{*}(t)$ and condition (3)(iv), we obtain $b\left(\left\|x^{*}(t)\right\|\right) \geq V\left(t, x^{*}(t)\right) \geq v\left(t ; t_{0}, v_{0}\right)>\varepsilon_{2}>b\left(\varepsilon_{3}\right), t \geq t_{0}$. Therefore $\left\|x^{*}(t)\right\|>\varepsilon_{3}$ for $t \geq t_{0}$.

Theorem 2 Let the following conditions be satisfied:

(1) The functions $g_{i} \in C\left[\mathbb{R}_{+} \times \mathbb{R}, \mathbb{R}\right], g_{i}(t, 0) \equiv 0, i=1,2$, and satisfy condition (H1).

(2) There exists a function $V_{1} \in \Lambda\left(\mathbb{R}_{+}, B(A)\right)$ such that

(i) the inequality

$$
{ }_{(4)}^{c} D_{+}^{q} V_{1}\left(t, x ; t_{0}, x_{0}\right) \leq g_{1}\left(t, V_{1}(t, x)\right)
$$

holds for any $t_{0}, t \in \mathbb{R}_{+}, t \geq t_{0}$ and $x, x_{0} \in B(A)$, where $A>0$ is a given number;

(ii) $a(\|x\|) \leq V_{1}(t, x) \leq b(\|x\|)$ for $t \in \mathbb{R}_{+}, x \in B(A)$, where $a, b \in \mathcal{K}$.

(3) There exists a function $V_{2} \in \Lambda\left(\mathbb{R}_{+}, B(A)\right)$ such that

(iii) the inequality

$$
{ }_{(4)}^{c} D_{+}^{q} V_{2}\left(t, x ; t_{0}, x_{0}\right) \geq g_{2}\left(t, V_{2}(t, x)\right)
$$

holds for any $t_{0}, t \in \mathbb{R}_{+}, t \geq t_{0}$ and $x, x_{0} \in B(A)$; 
(iv) $c(\|x\|) \leq V_{2}(t, x) \leq d(\|x\|)$ for $t \in \mathbb{R}_{+}, x \in B(A)$, where $c, d \in \mathcal{K}$.

(4) The zero solution of the couple of FrDE (6) is uniformly strictly stable in couple.

Then the zero solution of the system FrDE (4) is uniformly strictly stable.

Proof Let $\varepsilon_{1} \in(0, A]$ be an arbitrary number and $t_{0} \in \mathbb{R}_{+}$be an arbitrary initial time. From condition (4) there exists $\delta_{1}=\delta_{1}\left(\epsilon_{1}\right)>0$, and for any $\delta_{2} \in\left(0, \delta_{1}\right]$ there exists $\epsilon_{2} \in\left(0, \delta_{2}\right]$ such that the inequalities $\left|u_{0}\right|<\delta_{1}$ and $\delta_{2}<\left|v_{0}\right|$ imply

$$
\left|u\left(t ; t_{0}, u_{0}\right)\right|<a\left(\epsilon_{1}\right), \quad t \geq t_{0}
$$

and

$$
\epsilon_{2}<\left|v\left(t ; t_{0}, v_{0}\right)\right|, \quad t \geq t_{0},
$$

where the couple of functions $\left(u\left(t ; t_{0}, u_{0}\right), v\left(t ; t_{0}, u_{0}\right)\right)$ is a solution of the IVP for $\operatorname{FrDE}(6)$.

Choose $\delta_{3} \in(0, A)$ such that $b\left(\delta_{3}\right)<\delta_{1}$. Choose $x_{0} \in \mathbb{R}^{n}$ with $\left\|x_{0}\right\|<\delta_{3}$, and let $x^{*}(t)=$ $x\left(t ; t_{0}, x_{0}\right)$ be the solution of the IVP for FrDE (4) for the initial data $\left(t_{0}, x_{0}\right)$.

Let $u_{0}=V_{1}\left(t_{0}, x_{0}\right)$. Let $u\left(t ; t_{0}, u_{0}\right)$ be the maximal solution of the first equation of the couple of FrDE (6). According to condition (2)(ii) and the choice of $x_{0}$, we obtain $u_{0}=$ $V_{1}\left(t_{0}, x_{0}\right) \leq b\left(\left\|x_{0}\right\|\right)<b\left(\delta_{3}\right)<\delta_{1}$. Therefore the first component $u\left(t ; t_{0}, u_{0}\right)$ of the solution of (6) satisfied (34).

Assume that the inequality

$$
\left\|x^{*}(t)\right\|<A \quad \text { for } t \geq t_{0}
$$

is not true. Then there exists a point $t^{*}>t_{0}$ such that $\left\|x^{*}(t)\right\|<A$ for $t \in\left[t_{0}, t^{*}\right)$ and $\left\|x^{*}\left(t^{*}\right)\right\|=A$. From Lemma 2 , for $\Delta=B(A)$ and $T=t^{*}$, we obtain

$$
V_{1}\left(t, x^{*}(t)\right) \leq u\left(t ; t_{0}, u_{0}\right) \quad \text { for } t \in\left[t_{0}, t^{*}\right] .
$$

From inequalities (37), (34) and condition (2)(i) we get

$$
a(A)=a\left(\left\|x^{*}\left(t^{*}\right)\right\|\right) \leq V_{1}\left(t^{*}, x^{*}\left(t^{*}\right)\right) \leq u\left(t^{*} ; t_{0}, u_{0}\right)<a\left(\varepsilon_{1}\right) \leq a(A) .
$$

The obtained contradiction proves that inequality (36) is true for $t \geq t_{0}$. Then Corollary 2 is applicable on $\left[t_{0}, \infty\right)$ and inequality (37) is satisfied not only on $\left[t_{0}, t^{*}\right]$ but also on $\left[t_{0}, \infty\right)$. According to condition (2)(ii) and inequality (36), we get $a\left(\left\|x^{*}(t)\right\|\right) \leq V_{1}\left(t, x^{*}(t)\right) \leq$ $u\left(t^{*} ; t_{0}, u_{0}\right)<a\left(\varepsilon_{1}\right)$. Therefore, for $t \geq t_{0}$, the inequality $\left\|x^{*}(t)\right\|<\varepsilon_{1}$ holds.

Let $\delta_{4} \in\left(0, \delta_{3}\right]$ be an arbitrary number. Then there exists $\delta_{5} \in\left(0, \delta_{4}\right]$ such that $c\left(\delta_{4}\right)>\delta_{5}$. According to the above, for $\delta_{5}$ there exists $\varepsilon_{2}^{*} \in\left(0, \delta_{5}\right]$ such that $\left|v_{0}\right|>\delta_{5}$ implies inequality (35), $\varepsilon_{2}=\varepsilon_{2}^{*}$, for the second component $v\left(t ; t_{0}, v_{0}\right)$ of the solution of (6). Let the initial value $x_{0} \in \mathbb{R}^{n}$ additionally satisfy $\left\|x_{0}\right\|>\delta_{4}$. From the above $x^{*}(t) \in B(A)$ for $t \geq t_{0}$. Choose $\varepsilon_{3} \in\left(0, \delta_{4}\right]$ such that $d\left(\varepsilon_{3}\right) \leq \varepsilon_{2}^{*}$.

Let $v_{0}=V_{2}\left(t_{0}, x_{0}\right)$ and let $v\left(t ; t_{0}, v_{0}\right)$ be the minimal solution of the second equation of the couple of FrDE (6). According to condition (3)(iv), it follows that $\left|v_{0}\right|=V_{2}\left(t_{0}, x_{0}\right) \geq$ $c\left(\left\|x_{0}\right\|\right)>c\left(\delta_{4}\right)>\delta_{5}$. Therefore, the component $v\left(t ; t_{0}, v_{0}\right)$ of the solution of FrDE (6) satisfies inequality (35) for $\varepsilon_{2}=\varepsilon_{2}^{*}$. 
From Corollary 2 we have

$$
V\left(t, x^{*}(t)\right) \geq v\left(t ; t_{0}, v_{0}\right) \quad \text { for } t \geq t_{0}
$$

From inequalities (39), (34) and condition (3)(iv) we get

$$
d\left(\left\|x^{*}(t)\right\|\right) \geq V\left(t, x^{*}(t)\right) \geq v\left(t ; t_{0}, v_{0}\right)>\varepsilon_{2}^{*} \geq d\left(\varepsilon_{3}\right) .
$$

Therefore, for $t \geq t_{0}$, the inequality $\left\|x^{*}(t)\right\|>\varepsilon_{3}$ holds.

Corollary 3 Let the conditions (2) and (3) of Theorem 2 be satisfied with $g_{i}(t, x) \equiv 0, i=1,2$.

Then the zero solution of the system of FrDE (4) is uniformly strictly stable.

Sufficient conditions for strict stability could be obtained in the case of one Lyapunov function.

Theorem 3 Let the following conditions be satisfied:

(1) The function $g_{i} \in C\left[\mathbb{R}_{+} \times \mathbb{R}, \mathbb{R}\right], g_{i}(t, 0) \equiv 0, i=1,2, g_{1}(t, u) \geq g_{2}(t, u)$ for $t \in \mathbb{R}_{+}$, $u \in \mathbb{R}$ and satisfies condition (H1).

(2) There exists a function $V \in \Lambda\left(\mathbb{R}_{+}, B(A)\right)$ such that

(i) the inequalities

$$
g_{2}(t, V(t, x)) \leq{ }_{(4)}^{c} D_{+}^{q} V\left(t, x ; t_{0}, x_{0}\right) \leq g_{1}(t, V(t, x))
$$

hold for any $t_{0}, t \in \mathbb{R}_{+}, t \geq t_{0}$ and $x, x_{0} \in \mathbb{R}^{n}$;

(ii) $a(\|x\|) \leq V(t, x) \leq b(\|x\|)$ for $t \in \mathbb{R}_{+}, x \in B(A)$, where $a, b \in \mathcal{K}, A>0$ is a given number.

(3) The zero solution of the couple of FrDE (6) is strictly stable (uniformly strictly stable) in couple.

Then the zero solution of the system $\operatorname{FrDE}(4)$ is strictly stable (uniformly strictly stable).

The result of Theorem 3 is a partial case of Theorem 1 and Theorem 2.

Competing interests

The authors declare that they have no competing interests.

Authors' contributions

Each of the authors RA, SH and DO contributed to each part of the work equally and read and proved the final version of the manuscript.

\section{Author details}

'Department of Mathematics, Texas A\&M University-Kingsville, Kingsville, TX 78363, USA. ${ }^{2}$ NAAM Research Group, King Abdulaziz University, Jeddah, Saudi Arabia. ${ }^{3}$ Plovdiv University, Tzar Asen 24, Plovdiv, 4000, Bulgaria. ${ }^{4}$ School of Mathematics, Statistics and Applied Mathematics, National University of Ireland, Galway, Ireland.

Acknowledgements

Research was partially supported by the Fund NPD, Plovdiv University, No. MU15-FMIIT-008.

Received: 29 September 2015 Accepted: 15 October 2015 Published online: 06 November 2015 
References

1. Agarwal, R, Hristova, S: Strict stability in terms of two measures for impulsive differential equations with 'supremum'. Appl. Anal. 91(7), 1379-1392 (2012)

2. Lakshmikantham, V, Devi, JV: Strict stability for impulsive differential systems. Nonlinear Anal., Theory Methods Appl. 21(10), 785-794 (1993)

3. Lakshmikantham, V, Mohapatra, RN: Strict stability of differential equations. Nonlinear Anal. 46(7), $915-921$ (2001)

4. Tian, YS, Liu, CG: Strict stability of impulsive differential equations. Acta Math. Sin. 22(3), 813-818 (2006)

5. Zhang, C, Fu, X: The variational Lyapunov function and strict stability theory for differential systems. Nonlinear Anal., Theory Methods Appl. 64(9), 1931-1938 (2006)

6. Lewandowski, R, Chorazyczewski, B: Identification of the parameters of the Kelvin-Voigt and the Maxwell fractional models, used to modeling of viscoelastic dampers. Comput. Struct. 88, 1-17 (2010)

7. Yu, F: Integrable coupling system of fractional soliton equation hierarchy. Phys. Lett. A 373, 3730-3733 (2009)

8. Li, CP, Zhang, FR: A survey on the stability of fractional differential equations. Eur. Phys. J. Spec. Top. 193, 27-47 (2011)

9. Aguila-Camacho, N, Duarte-Mermoud, MA, Gallegos, JA: Lyapunov functions for fractional order systems. Commun. Nonlinear Sci. Numer. Simul. 19, 2951-2957 (2014)

10. Baleanu, D, Sadati, SJ, Ghaderi, R, Ranjbar, A, Abdeljawad (Maraaba), T, Jarad, F: Razumikhin stability theorem for fractional systems with delay. Abstr. Appl. Anal. 2010, Article ID 124812 (2010)

11. Delavari, H, Baleanu, D, Sadati, J: Stability analysis of Caputo fractional-order nonlinear systems revisited. Nonlinear Dyn. 67(4), 2433-2439 (2012)

12. Duarte-Mermoud, MA, Aguila-Camacho, N, Gallegos, JA, Castro-Linares, R: Using general quadratic Lyapunov functions to prove Lyapunov uniform stability for fractional order systems. Commun. Nonlinear Sci. Numer. Simul. 22 650-659 (2015)

13. Hu, JB, Lu, GP, Zhang, SB, Zhao, L-D: Lyapunov stability theorem about fractional system without and with delay. Commun. Nonlinear Sci. Numer. Simul. 20, 905-913 (2015)

14. Li, Y, Chen, Y, Podlubny, I: Stability of fractional-order nonlinear dynamic systems: Lyapunov direct method and generalized Mittag-Leffler stability. Comput. Math. Appl. 59, 1810-1821 (2010)

15. Li, Y, Chen, Y, Podlubny, I: Mittag-Leffler stability of fractional order nonlinear dynamic systems. Automatica 45 , 1965-1969 (2009)

16. Devi, JV, Mc Rae, FA, Drici, Z: Variational Lyapunov method for fractional differential equations. Comput. Math. Appl. 64, 2982-2989 (2012)

17. Lakshmikantham, V, Leela, S, Devi, JV: Theory of Fractional Dynamical Systems. Cambridge Scientific Publishers, Cambridge (2009)

18. Lakshmikantham, V, Leela, S, Sambandham, M: Lyapunov theory for fractional differential equations. Commun. Appl. Anal. 12(4), 365-376 (2008)

19. Agarwal, R, O'Regan, D, Hristova, S: Stability of Caputo fractional differential equations by Lyapunov functions. Appl. Math. (accepted)

20. Podlubny, I: Fractional Differential Equations. Academic Press, San Diego (1999)

21. Samko, G, Kilbas, AA, Marichev, OI: Fractional Integrals and Derivatives: Theory and Applications. Gordon \& Breach, New York (1993)

22. Das, S: Functional Fractional Calculus. Springer, Berlin (2011)

23. Diethelm, K: The Analysis of Fractional Differential Equations. Springer, Berlin (2010)

24. Baleanu, D, Mustafa, OG: On the global existence of solutions to a class of fractional differential equations. Comput. Math. Appl. 59, 1835-1841 (2010)

25. Yakar, C, Gucen, MB, Cicek, M: Strict stability of fractional perturbed systems in terms of two measures. In: Baleanu, D, et al.(eds.) Fractional Dynamics and Control, vol. 10, pp. 119-132. Springer, Berlin (2012)

\section{Submit your manuscript to a SpringerOpen ${ }^{\circ}$ journal and benefit from:}

- Convenient online submission

Rigorous peer review

- Immediate publication on acceptance

- Open access: articles freely available online

- High visibility within the field

- Retaining the copyright to your article 